\title{
To FACC or not to FACC ??
}

\section{Bouchard $\cdot$ F. Pigot $\cdot$ L. Abramowitz}

(C) Lavoisier SAS 2019

Fistula Anal Crohn Closure, nom de code FACC... est la première étude de stratégie médico-chirurgicale dans la prise en charge des fistules anales de la maladie de Crohn et c'est un travail... français !

Actuellement soumis à une revue européenne, présenté à l'ECCO 2019 et aux JFHOD 2019 par son concepteur et investigateur principal, le Docteur Laurent Abramowitz, cosignataire de cet éditorial. Cette étude nationale multicentrique contrôlée randomisée compare l'obturation chirurgicale (colles, lambeaux et autres techniques visant à interrompre le trajet sans couper de sphincter) au simple retrait du séton pour les fistules anales de la maladie de Crohn, préalablement drainées par séton et contrôlées par un anti-TNF (en l'occurrence de l'adalimumab, en mono ou combothérapie) [1].

Le résultat n'est pas significativement différent entre obturation ou retrait simple : à 12 mois, rémission maintenue chez $56 \%$ (18/32 patients) après obturation, versus $65 \%$ (17/26 patients) après simple ablation du séton. Sans différence entre les fistules simples ou complexes (71\% vs $63 \%$ pour les fistules simples et $52 \%$ vs $67 \%$ pour les fistules complexes), les résultats de FACC plaident donc pour un traitement non interventionnel pour la majorité des malades, et d'autres alternatives sont à évaluer pour améliorer ces résultats.

\section{Quelles leçons tirer de cette étude de stratégie de prise en charge des fistules anales de la maladie de Crohn?}

\section{L'idée d'un progrès ?}

L'usage jusqu'au-boutiste de la chirurgie n'était-il justifié que par l'inefficacité des anciens traitements médicaux ?

D. Bouchard $(\bowtie) \cdot$ F. Pigot

Unité de proctologie, hôpital Bagatelle,

203, route de Toulouse, F-33400 Talence, France

e-mail : proctobouc@gmail.com

L. Abramowitz

Unité de proctologie, hôpital Bichat-Claude Bernard, AP-HP,

46, Rue Henri Huchard F-75018 Paris, France
Une association médico-chirurgicale intelligente peut aider ces malades : chirurgie de drainage ciblée associée à un traitement médical performant.

L'adalimumab n'est peut-être pas si mauvais que cela pour les fistules ano-périnéales de la maladie de Crohn, bien qu'aucune comparaison ne soit encore possible avec les autres anti-TNF...

Le retrait simple du séton permettrait de faire l'économie d'un geste chirurgical avec son intérêt financier et humain.

\section{Une complexification du problème ?}

Il n'est peut-être pas indispensable de boucher une fistule pour qu'elle guérisse, tout au moins dans la maladie de Crohn.

La prise en charge de cette maladie pourrait sembler se simplifier. En fait, le premier temps de drainage chirurgical a plus que jamais son importance et doit encore être effectué dans des centres experts avec une évaluation radiologique précise.

L'importance du drainage et la bonne synchronisation avec le traitement médical sont des points essentiels et incontournables de la prise en charge de ces lésions.

Il faudra savoir partager la responsabilité des échecs du traitement des fistules entre la chirurgie et le traitement médical.

Enfin, de nouvelles questions émergent : quand enlever le séton? quels sont les facteurs pronostiques?

Au final, cette étude FACC a confirmé le fait que simplifier la prise en charge de ces malades complexes n'est pas une erreur, si tant est qu'elle soit effectuée dans des centres spécialisés.

La portée de ce résultat peut être pondérée par les limites de l'étude : nombre de malades inclus inférieur à celui prévu, possible effet centre lié à une hétérogénéité du nombre d'inclusions.

Ainsi, en 2019, certaines questions demeurent quant à la prise en charge optimale des fistules ano-périnéales de la maladie de Crohn :

- Comment évaluer la réponse au traitement ? Il faut aujourd'hui exiger un score composite clinico-radiologique (critères cliniques de Présent et critères IRM). 
- Quels sont les critères IRM pertinents ? Une approche récente a été proposée par la modification du score de van Assche par Samaan [2].

- Dans quel délai évaluer la réponse au traitement, et au bout de combien de mois parle-t-on d'échec thérapeutique?

- À partir de quels éléments définir un échec du traitement médical ? ou un échec du traitement chirurgical ? Le groupe d'experts, qui a élaboré les algorithmes d'aide à la prise en charge pratique des lésions ano-périnéales de la maladie de Crohn en 2018 sous l'égide de la SNFCP, a essayé d'apporter des éléments de réponse en ce sens [3].

- Comment traiter au mieux une fistule de Crohn bien drainée et bien contrôlée par traitement médical ? Y at-il encore une place pour l'obstruction des fistules ? Si oui, avec quelle technique ?

Il reste encore à évaluer le devenir des malades en non réponse primaire aux anti-TNF, dont la suppuration reste active malgré un bon drainage initial et un traitement par anti-TNF...

Liens d'intérêts : D. Bouchard est consultant pour les laboratoires AbbVie, Janssen et Takeda ; F. Pigot et L. Abramowitz sont consultants pour le laboratoire AbbVie.

\section{Références}

1. https://www.ecco-ibd.eu/publications/congress-abstract-s/item/ p560-multi-centric-randomised-study-comparing-interventional-vsnon-interventional-treatment-for-anal-fistulas-in-patient-withcrohn-8217-s-disease-and-adalimumab-treatment.html

2. Samaan MA, Puylaert CAJ, Levesque BG, et al (2017) The developpement of a magnetic resonance imaging index for fistulising Crohn's disease. APT 46:516-28

3. Bouchard D, Pigot F, Staumont G,et al (2018) Management of anoperineal lesions in Crohn's disease: a French National Society of Coloproctology national consensus. Tech Coloproctol 22:905-17 